\title{
Cosmic Rays from PeV to ZeV, Stellar Evolution, Supernova Physics and Gamma Ray Bursts
}

\author{
Peter L. Biermann ${ }^{1,2}$, Sergej Moiseenko ${ }^{3,1}$, Samvel Ter-Antonyan ${ }^{4,1}$, \& Ana \\ Vasile $^{1,5}$
}

${ }^{1}$ Max-Planck Institut für Radioastronomie, Bonn, Germany

2 Department for Physics and Astronomy, University of Bonn

${ }^{3}$ Space Research Institute, Moscow, Russia

4 Yerevan Physics Institute, Erewan, Armenia

${ }^{5}$ Institute for Space Sciences, Bucuresti-Magurele, Romania

plbiermann@mpifr-bonn.mpg.de,moiseenko@mx.iki.rssi.ru, samvel@jerewan1.yerphi.am,avasile@venus.nipne.ro

\author{
www.mpifr-bonn.mpg.de \\ /div/theory/ \\ www.mpifr-bonn.mpg.de/imprs/ \\ www.physik-astro.uni-bonn.de
}

\section{Abstract}

The recent success of a proposal from some time ago to explain the spectrum of cosmic rays allows some strong conclusions to be made on the physics of supernovae: In the context of this specific proposal to explain the origin of cosmic rays, the mechanism for exploding supernovae of high mass has to be the one proposed by Bisnovatyi-Kogan more than 30 years ago, which was then based on a broader suggestion by Kardashev: A combination of the effects of rotation and magnetic fields explodes the star. Interestingly, this step then leads inevitably to some further suggestions, useful perhaps for the study of gamma ray bursts and the search of a bright standard candle in cosmology.

\section{Introduction}

Cosmic Ray physics has inspired us for many years, and has given rise to many interesting books and reviews, some of which are [40, 17, 37, 18.

Several burning questions exist in high energy astrophysics:

- What is the physics of the supernova explosion of massive stars? The most common idea to be explored over the last few decades is that the burst of neutrinos, certainly sufficient in energy, as shown by the supernova 1987A, 
is doing it. However, the detailed mechanism has yet to be worked out successfully.

- What is the origin of Galactic cosmic rays? What makes it possible for cosmic rays from Galactic sources to reach energies such as $310^{18} \mathrm{eV}$ ?

- What is the mechanism of Gamma Ray Bursts, and what is it's relation to supernovae?

- Is there a brighter standard candle possibly available for cosmology than the now so famous Supernovae type Ia?

Here, in this review, we wish to combine several recent advances made to give a tentative answer to all these questions, starting with the work on cosmic rays:

\section{The cosmic ray spectrum}

We wish to explain the entire spectrum of cosmic rays, and focus here on Galactic cosmic rays, so the energies up to about $310^{9} \mathrm{GeV}$. Some time ago we made a proposal to explain Galactic and extragalactic cosmic rays in six consecutive papers, 20, 21, 22, 85, 77, 78, The basic idea was to distinguish the different sites of supernovae into the lower mass stars, that explode into the interstellar medium, and the higher mass stars that explode into their own stellar wind. The basic data to explain are as follows, 96, 68.

- The overall spectrum is about approx. $E^{-2.7}$ until the "knee", which is a bend downwards at around $310^{15} \mathrm{eV}$

- approx. $E^{-3.1}$ beyond the knee

- a slight downward dip from $310^{17} \mathrm{eV}$, sometimes referred to as the "second knee"

- a transition near $310^{18} \mathrm{eV}$, with then approx. $E^{-2.7}$ again

- uncertainty beyond $510^{19} \mathrm{eV}$, either a mild cutoff (HIRES) or a continuation (AGASA)

and in more detail, considering electrons and nuclei:

- electrons $E^{-2.7}$ as well (from radio, low $E$ ), up to about $20 \mathrm{GeV}$

- electrons $E^{-3.3}$ observed to $3 \mathrm{TeV}$ :

loss dominated, so injection $E^{-2.3}$

- positron fraction a few percent

- abundances enriched

- anti-proton fraction about $10^{-4}$

- heavy elements tend to have a slightly flatter spectrum 


\section{The arguments}

Already in 1934 the original suggestion by W. Baade \& F. Zwicky was that the most energetic particles probably come from the most energetic phenomonea known to us at the time, supernovae, [15]. E. Fermi then provided an argument on how reflection at magnetic irregularities can enhance the energy of a charged particle, 1949 and 1954, 34, 35. The detailed physics of the Fermi-acceleration was worked out in a simple approximation in a series of papers, starting in 1977 by I. Axford et al., G. Krymsky, [58, R. Bell in two papers, [16], and then again by R. Blandford \& J. Ostriker, 31] with a nice extensive review by L. Drury, 33.

\subsection{Supernova explosions into the ISM}

Then P. Lagage \& C. Cesarsky, 60], worked out the details for the maximum energy of a particle subject to Fermi acceleration in the shock of a supernova, exploding into the interstellar medium. Presciently, they already used the selfsimilar expansion into a very tenuous medium, now known to exist from X-ray observations. For the details of the acceleration of energetic particles at the shock they use the concept that most of the acceleration happens in a regime, where the magnetic field is parallel to the shock normal - as well its disturbances - and provides most of the scattering. Here the expansion solution is a similarity or Sedov-solution, a self-similarity, where the outer shock radius $r$ behaves as

$r \sim t^{2 / 5}$, and the shock speed is $\dot{r} \sim r^{-3 / 2}$, and so slows down considerably over time. This slow late evolution makes these supernova remnants dominant in observations, they just live a rather long time.

- $E_{\text {max }} \simeq 10^{5} Z B_{-6} \mathrm{GeV}$, using already the hot ISM of density $310^{-3} \mathrm{~cm}^{-3}$, confirmed only in 1997, 84]. The expansion is self-similar, usually referred to as a Sedov expansion, and yields then a maximum particle energy of about $Z 10^{14} \mathrm{eV}$, an order of magnitude below the knee.

- Maybe secondary acceleration is then required in the ISM - maybe by shockwaves inside superbubbles, the motley mixture of powerful stellar winds, and young supernova explosions. This picture has mostly been explored by I. Axford. A quantitative prediction is still outstanding for this scenario.

\subsection{The knee}

The key problem, recognized immediately, is that this maximum energy does not even come to the energy at the knee, and so that feature cannot be explained in such an approach. Therefore many attempts have been made: 


\subsubsection{Transport?}

Cosmic rays get injected with a spectrum, which is subsequently modified by losses from the disk, and so obviously, to get a kink in the spectrum may mean a change in behaviour of these diffusive losses; such a kink could be visible as the "knee". This is a question of the transport of cosmic rays through a turbulent magnetic ionized gas; general discussions of the magnetic field are in, e.g., [57, 59]. Such a change in transport implies a special scale in the interstellar medium, and would also lead to an increased anisotropy at the kink. However, there is no such scale as yet recognized.

\subsubsection{Turbulence?}

The evidence from the interstellar medium and from plasma physics suggests - if described by an isotropic approximation with an eye towards transport of cosmic rays - that the spectrum is a Kolmogorov spectrum. There are three arguments in favour of using a Kolmogorov spectrum for the context of cosmic rays: First, the difference in behaviour between nuclei, and electrons, allows the exponent of the diffusive law to be limited by about 0.4 , so the energy dependence of the diffusion coefficient $\kappa$ is weaker than $\kappa \sim E^{0.4}$, 24]. Second, any steeper dependence would produce anisotropies at the higher energies, which are not seen, 20]. And, finally, any steeper dependence would seriously dilute the number of stars contributing at the higher energies, making any reasonable powerlaw fit to the data meaningless, despite the fact, that such fits work well to describe the data, [25, 26].

\subsubsection{Spallation?}

Cosmic rays interact, and in fact most isotopes of Lithium, Beryllium, and Bor, as well as the odd-Z elements, and the sub-iron elements arise from cosmic ray interaction, the destruction of nuclei in a spallation process, see M. GarciaMunoz et al., 38, 39]. Therefore, a change in this interaction may lead to a loss of nuclei at higher energy; the interstellar medium is discussed, with some emphasis on the Galactic Center region, e.g., in 63. However, when working through this idea, one finds that the column for interaction must be much higher than what is observed; in fact, from the abundances of all the spallation products, as well as positrons and anti-protons we have a fairly good idea what the interaction actually is, and it is not enough to provide such a drastic change. Furthermore, that data suggest today, that the "knee" is a feature in rigidity $E / Z$, and so plasma physical processes are implied.

\subsubsection{New source?}

Obviously, in some proposals we require a new source at $310^{18} \mathrm{eV}$, or maybe even at $10^{14} \mathrm{eV}$, and so one might realistically ask, whether a new source might not be necessary already at lower energies, if that source is supposed to work so well at higher energies. A new source is easiest to accomodate, if the new source, 
dominant at higher energy, has a flatter spectrum than the source dominant at lower energy; in such a case the transition is trivial, since the higher energy source just overtakes the lower energy source naturally in flux. However, if the transition is supposed to occur at or near the knee, then the higher energy source must have a steeper spectrum rather than a flatter spectrum, and so we require a multitude of strange coincidences: The higher energy source must have a peaked spectrum, peaking just when the lower energy source peters out, and so have the same flux at that energy, while not contributing at lower energy in any significant way. That is really hard to believe. So, it is then actually simpler, and has been suggested, that we use a new source from the start, and, e.g., adopt the point of view, that Gamma Ray Bursts produce all the cosmic rays, in work by C. Dermer (discussed in a talk at the Aspen meeting, January 2002). However, then we are back where we started, we still need an argument why we have a change in slope at a specific rigidity, the knee. Also, the expected flux of cosmic rays from Gamma Ray Bursts is nowhere near to what is necessary, [75, 76. However, Gamma Ray Bursts should produce cosmic rays, and the question is whether there is any way to ascertain their contribution, perhaps through high energy neutrinos. Another possible source could be the activity in the Galactic Center, 61, 64. However, there is no observational evidence at this time, that such a localized activity could be dominant at the location of the Solar system.

\subsubsection{Change in acceleration?}

Therefore, what remains, is more mundane perhaps, a small modification in acceleration of the existing cosmic rays. The proposal by I. Axford et al. is that normal supernovae provide cosmic rays to about the knee, and that then in the supernova wind bubbles around the really massive stars, the cosmic rays get further acceleration, and then have a slightly diminished efficiency in acceleration; after all, the detailed physical properties are somewhat different in the environment of many supernovae and their predecessor winds, as compared to the interstellar medium. The only step missing in this very nice argument is a quantitative prediction as to what expect in this picture; one may surmise that the predictions may not turn out to be quite so different from the proposal described below.

\section{Proposal for the Origin of Galactic Cosmic Rays}

The riddle, which provoked a new approach now some time ago, was the key observation, that the radio shells of supernova remnants show a radial direction for the magnetic field, parallel to the shock-normal. This is in exact contradiction to the simple plasma physics expectation that the enhancement of the magnetic field component parallel to the shock surface should make that component dominant behind the shock, see [22].

Additional support for a new approach came from the observation that radio features could be seen in motion, with an amplitude of velocity quite close to 
the overall expansion speed of the supernova shock front, and yet measured with respect to the expanding frame of reference.

Third, the thickness of the observed shells both in the radio and also to some degrees in X-rays, is much too large to allow a simple explanation. There must be some reason for these shells to be almost $1 / 3$ of the outer radius oberved.

Finally, there appeared a theoretical argument, that cosmic ray dominated shocks are unstable, in work by R. Ratkiewicz, I. Axford, G. Webb, e.g., 79].

Therefore, we introduced the key premise that the particle transport in the shock region is dominated by large scale turbulence; in such a case there is no time for any turbulent cascade, and so no time for a Kolmogorov cascade either. Therefore the transport is just given by a specific scale, and a specific velocity, naturally the thickness of the shocked region, and the velocity difference across the shock. This is in the spirit of the early work on turbulence such as by L. Prandtl and Th.v. Karman. This premise allows then to explain all these observed features.

This means that the shock region is strongly turbulent, and the front of the shock, as seen along the line of sight at the edge of some observed supernova remnannt, is actually a superposition of many smaller shocks, advancing and retreating, like the waves on a beach. The geometric scale of these shocks is again given by the radial overall shock thickness, just given by momentum conservation. Therefore, curvature drifts do play a role, and introduce a scale in energy per charge $E / Z$.

It can be surmised, that the limit cycle arguments by M. Malkov \& L. O'C. Drury will eventually lead to a similar conclusion, 62. Such a convergence of theoretical concepts will be an important test of the picture.

\subsection{Supernovae into a stellar wind}

The concept then leads to the consideration of supernovae exploding into their own stellar wind, [93, 20, 21]. Stellar winds may be considered in a first approximation as a Parker wind, with $B_{\phi} \sim \sin \theta / r$ in spherical coordinates. The dominant magnetic field character is an Archimedian spiral, with the field mostly very nearly tangential. The key aspects are i) that the wind is magnetic, with the magnetic field given by the star, and not the interstellar medium, and ii) that the wind has a density gradient $\sim r^{-2}$, which means that the shock stays fast, and does not slow down as in an explosion into a medium of constant density. Also, magnetic fields in massive stars exist, they have been detected through nonthermal radio emission and maser emission, [21.

As the magnetic fields get very weak and also radial towards the pole of a Parker-type wind, one needs to consider shock acceleration in two limiting regimes:

At the pole and near to it we need to use the acceleration dominated by the magnetic field parallel to the shock normal, as done by 60]. However, at the equator, where the shock direction is perpendicular to the dominant magnetic field, we need to use another limit, as shown by R. Jokipii, [54]. Therefore we 
also need a matching condition, and this leads to a proposal for identifying the knee as arising with this matching energy, the maximum energy near the pole and close to it:

$$
E_{\text {max }}=\operatorname{Zer} B\left(3 / 4 v_{s h} / c\right)^{2}
$$

The maximum energy in the equatorial region, and in fact, most of $4 \pi$ is

$$
E_{\max }=Z \operatorname{er} B
$$

Since $B$ is inversely proportional to radius $r$ in the wind, these energies are actually constant throughout the wind, see e.g. [82. We still need to show, that the particles actually have enough time to reach these maximum energies, but this appears plausible. We also need to show, why this leads to a different spectrum beyond the knee: This is due to the effect that curvature drifts, which provide a small proportion of the particle's energy gain, when subject to many shock transitions, get slightly weaker when the Larmor radius of the particle moves past the scale corresponding to the maximum energy (really $E / Z$ ) near the pole, see [23] for a very detailed discussion.

Those stars which have powerful winds, are usually enriched in chemical elements such as Helium, Carbon and Oxygen, and so this line of reasoning actually picks up a theme, that Wolf Rayet stars are a major contributor to cosmic rays, 83. Therefore, supernova explosions into stellar winds are proposed to provide most of the Galactic cosmic rays, with the notable exception of the element Hydrogen, for which they provide only some fraction.

\section{Stellar evolution}

For the physics of cosmic rays and supernovae we need to consider stellar evolution in some detail. What happens to stars of different zero-age main sequence masses $M$ :

- Single stars with $M<8 M_{\odot}$ give no supernovae.

- Stars in the mass range $8<M<15 M_{\odot}$ explode as supernovae, but the explosion goes into the interstellar medium. Such explosions lead a Sedov type expansion, which becomes slow and has a long-lasting remnant.

- Stars with $15<M<30 M_{\odot}$ explode as supernovae with a substantial wind, but enriched only in Helium. The mass in the wind-shell is moderate. We refer to these stars as Red Super Giant stars (RSG).

- Stars $>30 M_{\odot}$ explode as supernovae into a strong wind; the chemical abundances are strongly enriched, since the mass loss eats deeply down back into the star. The mass in the wind-shell is large. We refer to these stars as Wolf-Rayet stars (WR).

Obviously, these numbers for the masses are approximations. 


\section{Predictions}

The predictions, all made some time ago, here focussing on wind-SNe, were described in [20, 21, 22, 85, 23, 24].

They gave the following quantitative spectra:

- $E^{-2.74 \pm 0.04}$ for the interstellar medium supernovae, with the abundances that correspond to the medium through which the supernova shock races. This spectrum is predicted to have a cutoff in the $100 \mathrm{TeV}$ range.

- $E^{-2.67-0.02 \pm 0.02}$ below knee for the wind supernovae, for which the shock races through the predecessor wind, and correspondingly has enriched abundances.

- $E^{-3.07-0.07 \pm 0.07}$ above knee for those same stars winds.

- A bending of the wind spectra at the "knee" near $Z 10^{15} \mathrm{eV}$, and a final cutoff at the "ankle" near $Z 310^{17} \mathrm{eV}$. These specific rigidities have an uncertainty of about a factor of 2 .

- When the supernova explodes, a powerful shock wave races through the wind, and then smashes into the shell; this wind-shell is made up of both wind-material and interstellar medium material, from the environment of the predecessor star. Such a shell has two shocks at its boundaries, one slowing down the wind, and one speeding up the interstellar material. If there is no convective instability, then a contact discontinuity separates wind material from environmental interstellar medium material.

- The shock is loaded with energetic particles from cosmic ray acceleration, and then these cosmic rays suffer from spallation in the shell, [27, 28]. Both cosmic ray particles as well as wind material particles get broken up. This, in the case of WR shells, gives the Be, B, Li nuclei in cosmic rays, which serve then as tracers of cosmic ray lifetimes, and interaction. It also gives the odd- $Z$ elements, as well as the sub-Fe elements in cosmic rays.

- As a new prediction following from the work in 28] we find a small spectral flattening is expected due to differential spallation for Fe-like nuclei. The fraction of nuclei removed by spallation from the set of Fe-like nuclei is so large, that across the range of energy per particle measured, this fraction is larger for lower energy, and so the spectrum flattened. This proposal is quantitatively consistent with the data.

At that stage we did not differentiate the cosmic ray acceleration scenario for red supergiant winds (RSG), and Wolf Rayet (WR) winds. We now do differentiate those two kinds of stars with winds in their interaction in the wind shell, 28. The RSG stars have a weaker wind, therefore a lower mass shell as compared to the WR stars, and so we argued that the cosmic ray interaction, when the supernova shock finally hits the shell, is convective for 
RSG stars, and diffusive for WR stars. In this picture the RSG stars produce the gamma ray emission from the Galactic plane, and the WR stars produce the spallation products such as Beryllium, and give only some small contribution to the Galactic gamma ray emission at moderate photon energies.

\subsection{Implications}

Such a model implies, as had been predicted already by B. Peters in the fifties, [71, 172, that the turnover at the knee, and at the final cutoff is gradual, as the elements roll off in sequence of $Z$. Furthermore, since the knee and final cutoff energy are given by stellar parameters, the magnetic field, implicitly the rotation (the wind must be asymptotic already at the surface, which implies slow rotation at the surface) and the explosion energy, these parameters should be very nearly the same for all stars.

This corresponds exactly to what has been argued in a very different context already by G. Bisnovatyi-Kogan in (1970), who based his argument in turn on a broader suggestion by N. Kardashev (1964), 29, [56]. In this picture, the predecessor star is rotating and has a magnetic field. When the core of the star collapses, the core is spun up to a fast rotating disk, and the collapse along the plane of symmetry stops. The potential energy at that stage then is transmitted to the envelope by the torque of the magnetic fields (i.e. their angular momentum transport), and that potential energy constitutes the explosion energy.

Therefore, if the data are really well fit by the model, the conclusion is strongly suggested that not only the concept of magneto-rotational explosion works, but that in fact it works with very similar numbers in all stars.

\section{New data}

Since the original detailed quantitative predictions were made, new data have appeared, which provide very serious tests, e.g. [51]:

- At present the data set from the KASCADE experiment is the best across the knee region, and provides a first challenging test. As we show below they are very well fit by the data. Other data also show consistency, but with large error bars, and with unknown systematics still, so further conclusions can not be drawn from them.

- In paper 27] we predicted the energy dependence of the $\mathrm{B} / \mathrm{C}$ ratio, from interaction in the thick WR-star shells, as $E^{-5 / 9}$. A refined treatment was given in 28]. As V. Ptuskin then showed some time later, at the ICRC in Salt Lake City, 74, the best fit to the data gives an energy dependence of $E^{-0.54}$, which is quite consistent.

- In 1997 St. Hunter et al., [52, and M. Mori, 67, showed that the gamma ray emission from the Galactic plane could only be fitted by a cosmic ray spectrum, which was substantially flatter than the one observed. In 
27. 28. we suggested that the spectrum could be explained as interaction in the source region of cosmic rays, specifically the RSG star shells. The interaction happens then with a spectrum of $E^{-7 / 3}$. We will discuss the details of this fit elsewhere.

\subsection{Specific tests}

The tasks ahead of us are the following, to be quite specific:

- We normalize all abundances at $\mathrm{TeV}$, using the data collection of [96].

- We need to remember, that Hydrogen has two components, only one of which may have a knee (wind-SNe, RSG), the other one has a cutoff below the knee (ISM-SNe); on the other hand Hydrogen is extremely low in WR winds. We need to note here, that the data do not force us at this time to assume that the knee of the RSG stars and the WR stars is the same; in fact, it could be, that both knee and cutoff for RSG stars are quite different, much lower in rigidity than for WR stars. In our first data fits, described below, we do not yet distinguish RSG and WR stars as regards the knee and final cutoff. The KASCADE data are forceful only for the abundances from the WR stars.

- Also Helium may have two different observable knees or cutoffs, corresponding again to RSG supergiants, and WR supergiants, but they also might be same; we have to keep this uncertainty in mind.

- We then extrapolate with the predicted spectrum to the knee in rigidity, bend at the knee in rigidity, and extrapolate to the cutoff. As the cutoff is also in rigidity, there is a rolloff of the overall spectrum, starting from the cutoff in Helium, which we can actually identify with the "second knee".

- We then compare to KASCADE, Akeno, HIRES, ... One important point is to remember, that any fit to the data across the knee has to "reach" the data at higher energy, such as from Akeno, etc. This restricts any possible fits quite severely, see [51].

\subsection{Work to be done}

The work is spread among many of our partners, as follows:

- The detailed abundances are being considered with A. Popescu (Bukarest), and N. Langer (Utrecht).

- The energy in explosions is considered with G. Pavalas (Bukarest).

- The positron spectra are done with W. Rhode et al.(Wuppertal).

- The gamma ray spectrum of the Galaxy is done with S. Casanova (Bonn), R. Engel (Karlsruhe), W. Rhode (Wuppertal), and many others. 
- The anti-proton spectra are done with E.-S. Seo, R. Sina (Univ. Maryland), and R. Engel (Karlsruhe).

- The detailed fit to the KASCADE data is first described in this paper, in the following main section.

- Later we will also consider a more detailed fit to the Akeno, AGASA and HIRES near the ankle with S. Ter-Antonyan (coauthor here), A. Vasile (coauthor here), Zh. Cao (Utah) and St. Westerhoff (New York).

\section{Cosmic ray properties from air shower data}

The following section was written by S. Ter-Antonyan.

In general, the relation between energy spectra $\left(\partial \Im_{A} / \partial E_{0}\right)$ of primary nuclei $(A)$ and measured EAS size spectra at observation level $\left(\Delta I / \Delta N_{e, \mu}^{*}\right)$ is determined by an integral equation

$$
\frac{\Delta I(\theta)}{\Delta N_{e, \mu}^{*}}=\sum_{A} \int_{E_{\min }}^{\infty} \frac{\partial \Im_{A}}{\partial E_{0}} \frac{\partial W\left(E_{0}, A, \theta\right)}{\partial N_{e, \mu}^{*}} d E_{0}
$$

where the kernel function $\left(\partial W / \partial N_{e, \mu}^{*}\right)$ of the equation depends on primary energy, type of primary nucleus $(A=1,4, \ldots, 59)$, zenith angle $(\theta), A-A_{A i r}$ interaction model and response functions of measurements.

Eq. (3) is a typical ill-posed problem and has an infinite set of solutions for unknown primary energy spectra. However, the integral Eq. (3) turns to a Fredholm equation, if the type of a primary nucleus is defined directly in the experiment (as it is in the balloon and satellite measurements [12, 8] where energy spectra for different nuclei are obtained up to $10^{15} \mathrm{eV}$ ).

Similarly to [41, 91] we reconstruct primary energy spectra based on a transformation of the integral Eq. (3) to a parametric equation with unknown spectral parameters. For that, instead of unknown primary energy spectra of the integral Eq. (3) we consider energy spectra according to the predictions of a multi-component model [20, 24]. In this case, unknowns are such model parameters as cut-off and knee energies, spectral indices, scale factors and fractions of different components. EAS muon and electron size spectra on the left side of the Eq. (3) are taken from recent KASCADE publications [92, 43].

The kernel function of Eq. (3) $\partial W / \partial N_{e, \mu}$ is preliminarily calculated by a MonteCarlo method provided a given $\left(A-A_{A i r}\right)$ interaction model of primary nuclei and atmosphere.

Solutions of Eq. (3) for unknown spectral parameters at a priori known primary spectra are easy to obtain by means of $\chi^{2}$-minimization method [90, 91]. It is clear that solutions found that way are partial solutions of the integral Eq. (3). However, if the values of the spectral parameters are consistent with the predictions of a primary spectrum model calculations [20, it will confirm the use of this model in the investigation energy region.

We have already done such investigations 91 by testing a multi-component model of primary cosmic ray origin with observed EAS size spectra from KASCADE $\left(1020 \mathrm{~g} / \mathrm{cm}^{2}\right)$ and ANI $\left(700 \mathrm{~g} / \mathrm{cm}^{2}\right)$ using experiments in 5 different 
zenith angular intervals.

Here we consider the inverse problem based on KASCADE EAS electron and "truncated" muon size spectra 92, 43, in 3 zenith angular intervals. The combined analysis of electron and muon EAS size spectra allows us to determine the influence of a nuclear interaction model on the quality of the inverse problem solution more accurately.

\subsection{Parametrization of the primary energy spectra}

The energy spectra of primary nuclei according to the multi-component model of primary cosmic ray origin [20, 24] at energies $10^{12}-10^{18} \mathrm{eV}$ are presented here in a 3-component form:

$$
\frac{\partial \Im}{\partial E_{A}}=\beta \Phi_{A}\left(\delta_{A, 1} \frac{d \Im_{1}}{d E_{A}}+\delta_{A, 2} \frac{d \Im_{2}}{d E_{A}}\right)+\Phi_{A}^{E G} \frac{d \Im_{3}}{d E_{A}}
$$

where the $\beta$ is a dimensionless normalization parameter, $\Phi_{A}$ are scale spectral factors and model parameters $\delta_{A, i=1,2}$ are the fractions of each component $\left(\delta_{A, 1}+\delta_{A, 2}=1\right)$ in a primary flux of nuclei $(\mathrm{A})$.

The first component (ISM) is derived from explosions of a normal supernova into an interstellar medium with expected rigidity-dependent power law spectra

$$
\frac{d \Im_{1}}{d E_{A}}=\left\{\begin{array}{lll}
E_{A}^{-\gamma_{1}} & : & E_{A}<E_{c u t} Z \\
0 & : & E_{A}>E_{c u t} Z
\end{array}\right.
$$

where the model parameter $E_{\text {cut }} Z$ is a cut-off energy of ISM component at $Z$ nuclear charge.

The second component (SW) is a result of explosions of stars into their former stellar winds with expected rigidity-dependent power law spectra

$$
\frac{d \Im_{2}}{d E_{A}}= \begin{cases}E_{A}^{-\gamma_{2}} & : E_{A}<E_{k} Z \\ E_{k}^{-\gamma_{2}}\left(E_{A} / E_{k}\right)^{-\gamma_{3}} & : E_{A}>E_{k} Z \\ 0 & : E_{A}>E_{\text {cut }}^{S W} Z\end{cases}
$$

where the model parameter $E_{k} Z$ is a knee energy of SW component and $E_{c u t}^{S W} \simeq$ $2.2 \cdot 10^{5} \mathrm{TeV}$ is a corresponding cut-off energy [20].

The third, extragalactic (EG) component is approximated also by rigiditydependent power law spectra

$$
\frac{d \Im_{3}}{d E_{A}}= \begin{cases}E_{a n k}^{-2.75}\left(E_{A} / E_{a n k}\right)^{-2} & : E_{A}<E_{a n k} Z \\ E_{A}^{-2.75} & : E_{A}>E_{a n k} Z\end{cases}
$$

The values of model predictions [20] for spectral parameters are:

$\gamma_{1}=2.75 \pm 0.04, \gamma_{2}=2.67 \pm 0.03, \gamma_{3}=3.07 \pm 0.1, E_{\text {cut }} \simeq 120 \mathrm{TV}, E_{k} \simeq 700$ $\mathrm{TV}, \Phi_{A=1}^{E G} \simeq 0.032\left(\mathrm{~m}^{2} \cdot \mathrm{sec} \cdot \mathrm{ster} \cdot \mathrm{TeV}\right)^{-1}, E_{\text {ank }} \simeq 6.5 \cdot 10^{5} \mathrm{TV}$ at factors of uncertainty $\sim 2$.

In 91] we have already obtained the evaluations of spectral indices of ISM and SW components: $\gamma_{1}=2.78 \pm 0.03, \gamma_{2}=2.65 \pm 0.03$ as solutions of parametric 
Eq. (3) using KASCADE [42 and 32] EAS size spectra at 5 zenith angular intervals. This is in quite good agreement with a similar early test 85. Since these values agreed with the model predictions here we set them fixed.

\subsection{EAS size spectra}

In general, the kernel function of Eq. (3) in EAS inverse problems is determined by

$$
\frac{\partial W}{\partial N_{e, \mu}^{*}} \equiv \int_{\theta_{1}}^{\theta_{2}} \int_{0}^{\infty} \frac{\partial G\left(E_{0}, A, \theta\right)}{\partial N_{e, \mu}} \frac{\partial P\left(N_{e, \mu}\right)}{\partial N_{e, \mu}^{*}} \frac{\sin \theta}{\Delta_{\theta}} d \theta d N_{e, \mu}
$$

where $\partial G / \partial N_{e, \mu}$ is an expected EAS size electron (truncated muon) spectrum at a given observation level for $E_{0}, A, \theta$ parameters of a primary nucleus and depends on $A-A_{A i r}$ interaction model, $\Delta_{\theta}=\cos \theta_{1}-\cos \theta_{2}$, and $\partial P / \partial N_{e, \mu}^{*}$ is the error function of measurements 90 .

We calculated the shower spectra $\partial G / \partial N_{e, \mu}$ at KASCADE observation level $\left(1020 \mathrm{~g} / \mathrm{cm}^{2}\right)$ using the CORSIKA6016(NKG) EAS simulation code [45] with the QGSJET01 [55] and SIBYLL2.1 36] interaction models. Input parameters in simulations were: primary energies $E_{A} \equiv 32,100, \ldots 3.2 \cdot 10^{5} \mathrm{TeV}, 4$ groups of primary nuclei $A \equiv 1,4,16,56$ and 3 zenith angular intervals similarly to KASCADE experimental data 92, 43]. Intermediate values are calculated using log-linear interpolations. Relative statistical errors of calculated shower spectra were less than $3 \%$.

The expected detectable EAS size spectra $\partial W / \partial N_{e, \mu}^{*}$ were calculated using logGaussian approximations for $\partial G / \partial N_{e, \mu}$ and error functions $\partial P / \partial N_{e, \mu}^{*}$ which were obtained with high accuracy in the investigated energy range.

We also investigated the error function of measurements using CORSIKA code and for reconstructions of truncated muon sizes we obtained accuracy approximation: $\Delta N_{\mu} / N_{\mu} \simeq 15 / \sqrt{N}_{\mu}+0.05$ that exceeds the corresponding values of KASCADE approximation [41. In our calculations we used a more precise standard option of CORSIKA6016.

For the left side of Eq. (3) we used KASCADE EAS electron and truncated muon size spectra from 92, 43. (Fig. 2, symbols). In order to make a comparison and subsequent normalization of our data with direct balloon and satellite measurements at about $10^{14}$ energy range we also considered the data from early publications of KASCADE [42] with corresponding zenith angular corrections (the first 7 symbols for each angular interval in Fig. 22 ).

\subsection{Results}

Minimization of $\chi^{2}(\mathbf{I}, \mathbf{P})$-functional with a measurement vector

$$
\mathbf{I} \equiv\left\{\Delta I / \Delta N_{e, i, k}^{*}, \Delta I / \Delta N_{\mu, j, k}^{*}\right\}
$$

and a corresponding prediction vector $\mathbf{P}$ from the right-hand part of Eq. (3) was carried out at $i=1, \ldots, 42$ energy intervals of EAS electron size spectra, 


\begin{tabular}{ccccc}
\hline $\begin{array}{c}\text { Spectral } \\
\text { Parameters }\end{array}$ & QGSJET01 & SIBYLL2.1 & $\begin{array}{c}\text { 3-component } \\
\text { predictions }\end{array}$ & Comments \\
\hline$\gamma_{1}$ & $2.78 \pm 0.03$ & - & $2.75 \pm 0.04$ & {$[91]$} \\
$\gamma_{2}$ & $2.65 \pm 0.03$ & - & $2.67 \pm 0.03$ & {$[91]$} \\
$\gamma_{3}$ & $3.25 \pm 0.04$ & $3.25 \pm 0.04$ & $3.07 \pm 0.1$ & $3.28 \pm 0.07[91]$ \\
$E_{\text {cut }}$ & $200 \pm 100$ & $200 \pm 100$ & $120-250$ & $210 \pm 60 \mathrm{TV}[91]$ \\
$E_{k}$ & $2100 \pm 140$ & $1910 \pm 150$ & $700-1400$ & $1900 \pm 100 \mathrm{TV}[91]$ \\
$\delta_{A=1,2}$ & $0.47 \pm 0.04$ & $0.5 \pm 0.04$ & - & $0.5-0.8[91]$ \\
$\delta_{A>1,2}$ & 0.9 & 0.9 & - & $0.85-1[91]$ \\
$\beta \Phi_{P}$ & $0.120 \pm 0.007$ & $0.106 \pm 0.006$ & & $\left(\mathrm{~m}^{2} \cdot \mathrm{s} \cdot \mathrm{sr} \cdot \mathrm{TeV}\right)^{-1}$ \\
$\beta \Phi_{\mathrm{He}}$ & $0.089 \pm 0.011$ & $0.084 \pm 0.010$ & & $\left(\mathrm{~m}^{2} \cdot \mathrm{s} \cdot \mathrm{sr} \cdot \mathrm{TeV}\right)^{-1}$ \\
$\beta \Phi_{O}$ & $0.058 \pm 0.007$ & $0.064 \pm 0.006$ & & $\left(\mathrm{~m}^{2} \cdot \mathrm{s} \cdot \mathrm{sr} \cdot \mathrm{TeV}\right)^{-1}$ \\
$\beta \Phi_{F e}$ & $0.026 \pm 0.005$ & $0.035 \pm 0.005$ & & $\left(\mathrm{~m}^{2} \cdot \mathrm{s} \cdot \mathrm{sr} \cdot \mathrm{TeV}\right)^{-1}$ \\
\hline
\end{tabular}

Table 1: Spectral parameters of reconstructed energy spectra (446) for 4 groups of primary nuclei in the framework of a 3-component model of primary cosmic ray origin and QGSJET and SIBYLL interaction models based on KASCADE date.

$j=1, \ldots, 26$ energy intervals of EAS truncated muon size spectra and $k=1,2,3$ zenith angular bins from KASCADE data 92, 43. However, the combined analysis of electron and muon size spectra at $\chi^{2}$-minimization requires to include in the expected shower spectra 2 additional unknown dimensionless parameters $\eta_{e}$ and $\eta_{\mu}$ which define a constant bias of each spectrum due to peculiarities of interaction model and systematic measurement errors [90.

In the first instance, inner spectral parameters $\left(\gamma_{1,2,3}, E_{c u t}, E_{k}, \delta_{A}, \Phi_{A}\right)$ of primary spectra (4,6) have been evaluated using normalized dimensionless EAS size spectra in the measurement vector

$$
\mathbf{I}_{\mathbf{n}} \equiv\left\{\frac{1}{U_{e}} \frac{\Delta I}{\Delta N_{e, i, k}^{*}}, \frac{1}{U_{\mu}} \frac{\Delta I}{\Delta N_{m, j, k}^{*}}\right\}
$$

where the scalar parameters $U_{e}=\sum_{i, k}\left(\Delta I / \Delta N_{e, i, k}\right)$ and $U_{\mu}=\sum_{j, k}\left(\Delta I / \Delta N_{\mu, j, k}\right)$. Corresponding normalizations of spectra were performed for all components of the prediction vector $\mathbf{P}_{\mathbf{n}}$ at the minimization of the $\chi^{2}\left(\mathbf{I}_{\mathbf{n}}, \mathbf{P}_{\mathbf{n}}\right)$-functional. After evaluation of the inner spectral parameters the values of $\eta_{e}$ and $\eta_{\mu}$ were determined using the $\chi^{2}(\mathbf{I}, \mathbf{P})$ functional by definition (8) at fixed inner spectral parameters.

Finally, the dimensionless parameter $\beta$ in expression (44) was determined by a normalization of the all-particle spectrum obtained $\sum\left(\partial \Im / \partial E_{A}\right)$ with JACEE data at $100 \mathrm{TeV}$ energy.

Table 1 contains the values of all spectral parameters which were obtained by the method above at QGSJET01 [55] and SIBYLL2.1 [36] interaction models and KASCADE EAS data 92, 43. The normalization factors in Table 1 are: $\beta=1.13 \pm 0.05$ at QGSJET model and $\beta=1.0 \pm 0.05$ SIBYLL model.

The energy spectra obtained of different nuclei and the corresponding allparticle spectrum in comparison with JACEE [12] and RUNJOB [8] direct 
measurements and EAS data [86 up to energy $3 \cdot 10^{18} \mathrm{eV}$ are presented in Fig. 11.

The thick line corresponds to the expected energy spectra of the extragalactic component [20, 85, 77, 78]. This flux is also included in the all-particle spectrum in Fig. [1]

The expected EAS electron and truncated muon size spectra corresponding to our primary spectra obtained with the QGSJET interaction model in comparison with the KASCADE data 92, 43] are shown in Fig. 2a,b.

The value of $\chi^{2} \simeq 1$ with $2 \%$ uncertainty (the width of a shaded area) of expected data (and $4 \%$ uncertainty at SIBYLL model). For biases of shower spectra (Fig. 20 $\mathrm{a}, \mathrm{b}$ ) we obtained the values: $\eta_{e}=1.23 \pm 0.04, \eta_{\mu}=1.12$ at QGSJET model and $\eta_{e}=1.0 \pm 0.01, \eta_{\mu}=1.3 \pm 0.02$ at SIBYLL model. These values point out the possible existence of systematic biases both in the EAS measurements and in the interaction models.

In Fig. 3 the expected muon lateral distribution functions (symbols) and corresponding KASCADE data shaded area) from [4] at different intervals of detected truncated muon size are shown.

In order to test the behavior of primary energy spectra obtained in a larger energy range we also calculated the expected hadron energy spectra at mountain level for comparison with data of the PION experiment (3200m a.s.l.) [14, 89. at 1-7 TeV hadron energies at observation level $\left(700 \mathrm{~g} / \mathrm{cm}^{2}\right)$. The calculation was carried out using the QGSJET and SIBYLL interaction models and using the method introduced above without normalizations $(\beta=1)$. The best agreement of the expected hadron flux at mountain level in the framework of the 3-component primary model predictions and PION data was achieved at $\delta_{A=1,2}=0.35 \pm 0.05$ and $\delta_{A>1,2}=0.9 \pm 0.1$ fractions of component. These results are shown in Fig. 4. The effective primary energy range corresponding to these data is $E_{0} \sim 10-100 \mathrm{TeV} /$ nucleon.

Thus, predictions of the multi-component model of the cosmic ray origin 20. 24 explain the measured KASCADE EAS data in the knee region $\left(E_{0} \simeq\right.$ $\left.10^{14}-10^{17} \mathrm{eV}\right)$ and hadron spectra at mountain level $\left(E_{0} \simeq 10^{13}-10^{14} \mathrm{eV}\right)$ with an accuracy of $10-15 \%$ in the framework of the QGSJET and SIBYLL interaction models respectively.

The rigidity-dependent behavior of spectra for different primary nuclei is the same for the two interaction models.

The agreement of the expected all particle spectrum and world data in $E_{0} \simeq$ $10^{17}-10^{18} \mathrm{eV}$ primary energy range displays the presence of the extragalactic component of primary cosmic rays in accordance with the 3-component model predictions.

\section{The fit and consequences}

Here we have described the actual fit to the data; we did this in two steps, first just a direct fit to the KASCADE elemental data as published, as done by 


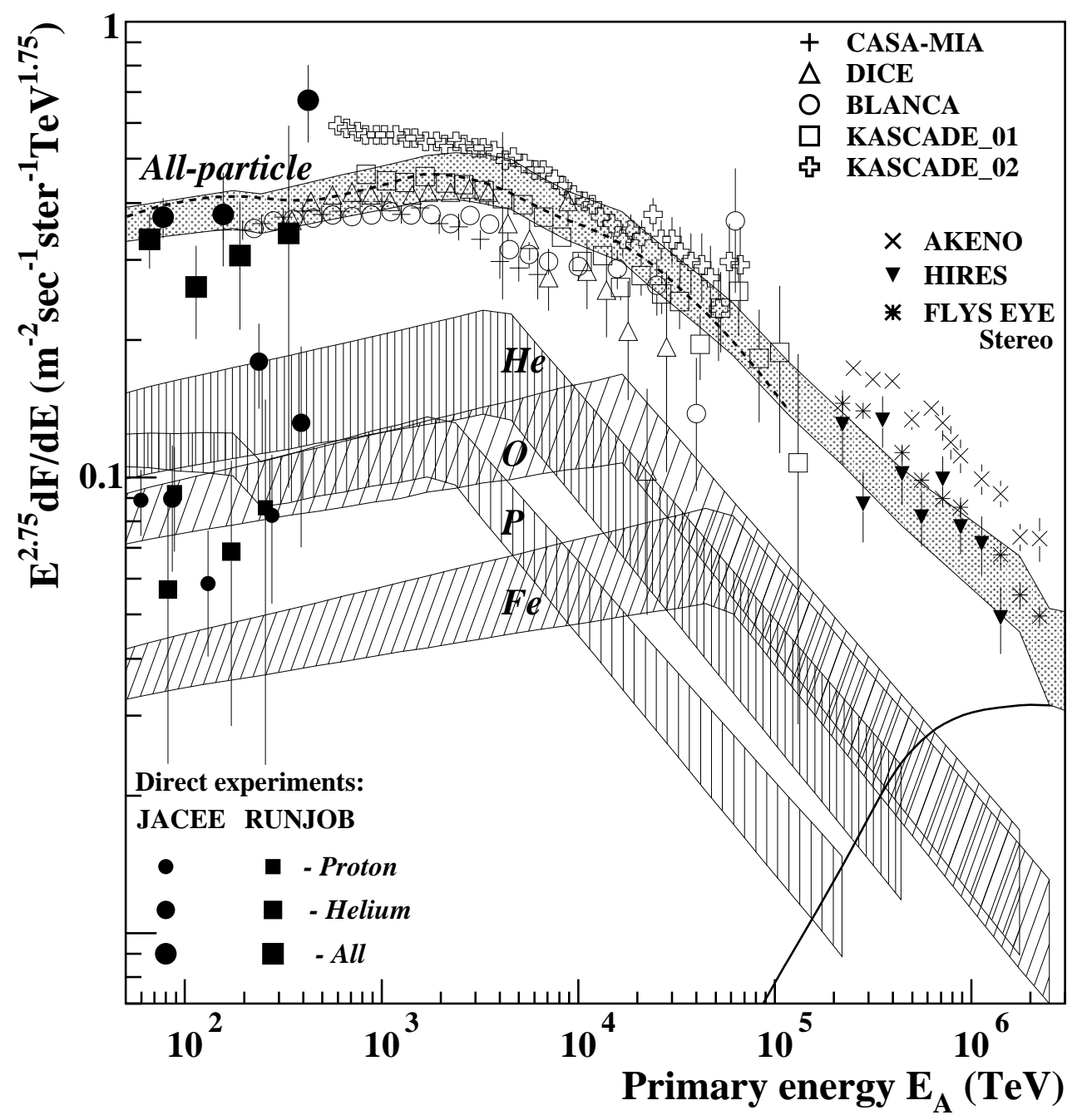

Figure 1: Expected all-particle energy spectrum and energy spectra of different nuclei (shaded areas) according to a 3 -component model of primary cosmic rays. The solid line is the expected (third) extragalactic component and the dashed lines correspond to the all-particle spectrum from 91. The JACEE, RUNJOB, CASA, DICE, BLANCA, KASCADE02 data is taken from the review [86], KASCADE01 - from 92. 

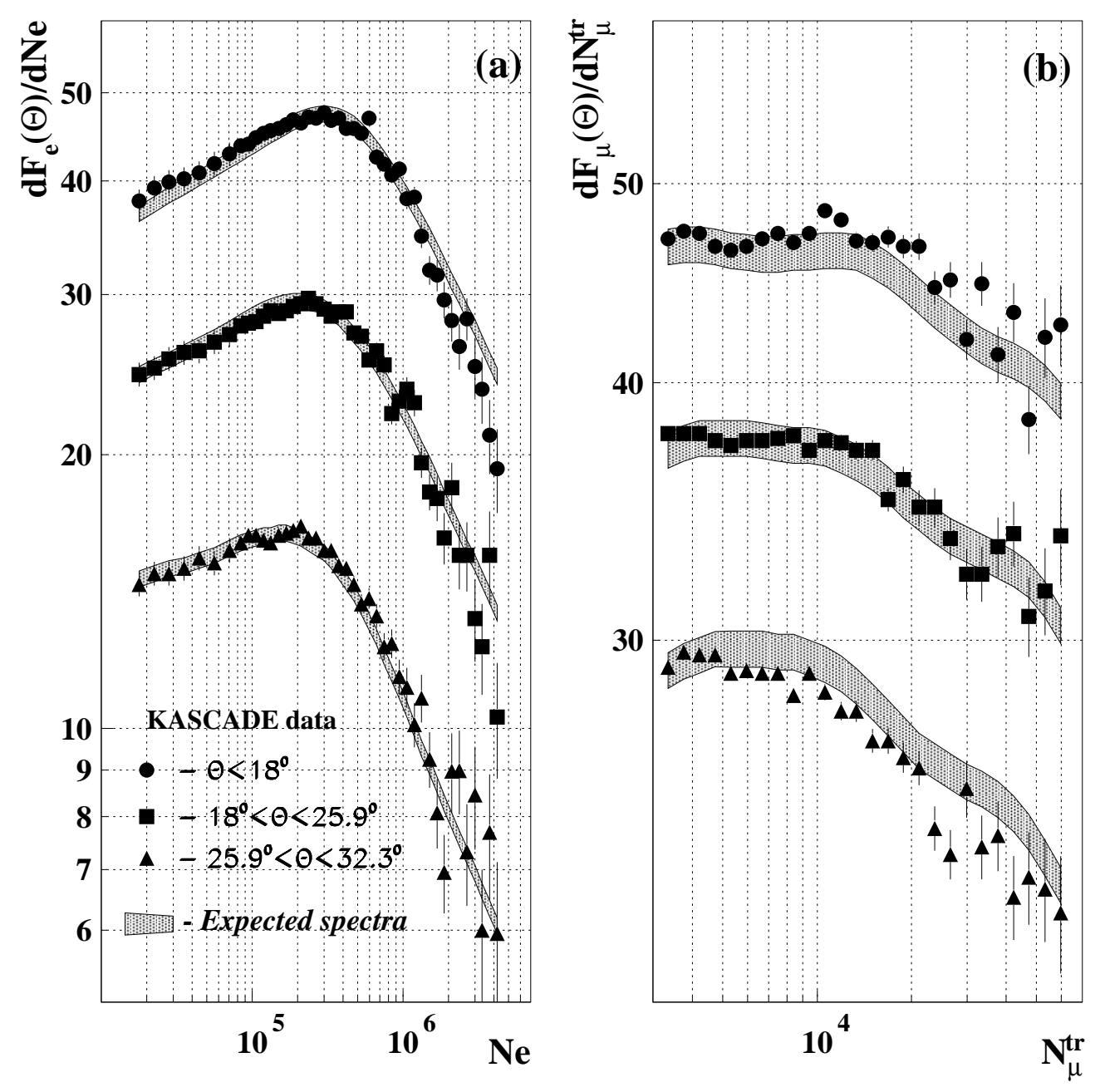

Figure 2: KASCADE EAS electron (a) and truncated muon (b) size spectra (symbols) at 3 zenith angular bins (from 92 ) and corresponding expected spectra (shaded areas) in the framework of a 3-component model of primary cosmic ray origin and the QGSJET interaction model. 


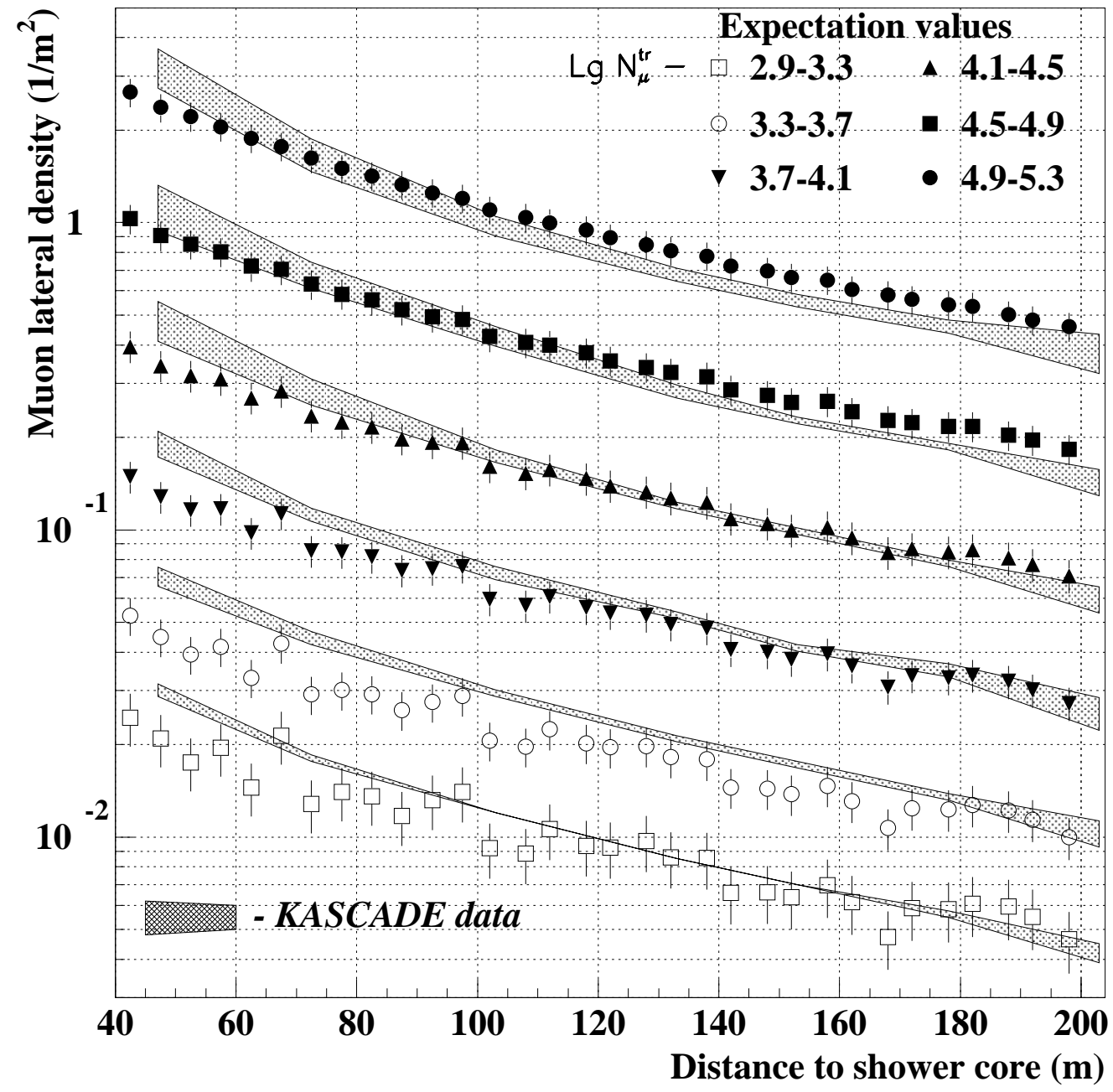

Figure 3: KASCADE muon lateral distribution functions (shaded areas) at different truncated muon sizes and the corresponding 3-component model predictions with the QGSJET interaction model (symbols). 


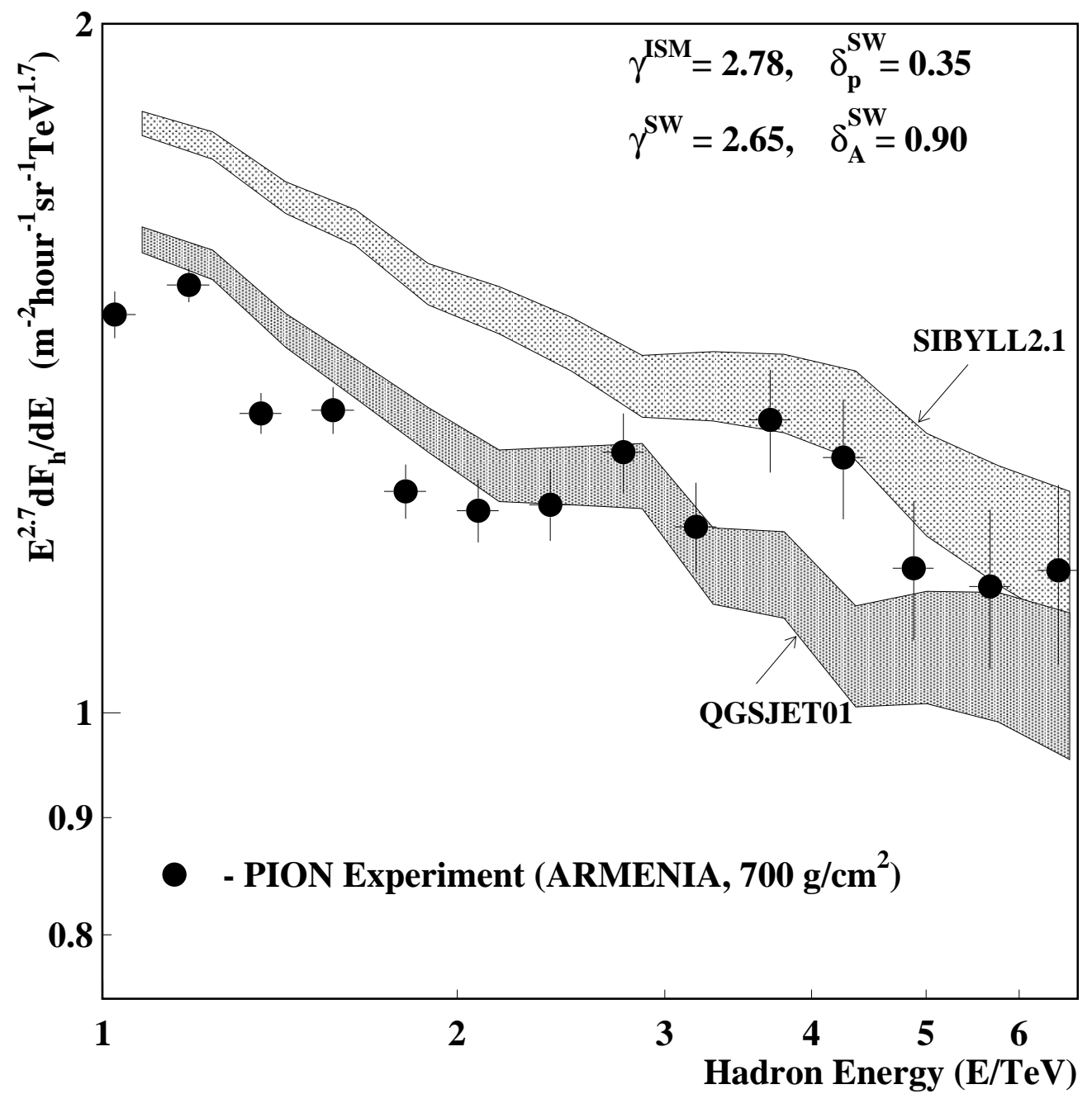

Figure 4: Vertical hadron energy spectrum at mountain level $\left(700 \mathrm{~g} / \mathrm{cm}^{2}\right)$ obtained by the PION experiment (symbols) and the expected spectra (a shaded areas) in the framework of the 3-component primary model and QGSJET and SIBYLL interaction models. 
A. Vasile, and then by an extensive Monte Carlo run to consider all published detailed distributions from KASCADE directly, described in the section above written by S. Ter-Antonyan. We refer here to the ICRC publications, such as [92, 50. An independent, but similar approach is described by G. Schatz, 81]; recent KASCADE papers are [5, 6, 51, 7]. It is gratifying, that a first direct fit, as done by A. Vasile, and the extensive fitting done by S. Ter-Antonyan, not only agree with each other, but actually agree with the prediction, and with the early tests, 85].

We summarize, that the KASCADE data as well as a first approximation to the HiRes, Akeno and Yakutsk data can be fully fitted by the model with parameters very close to what had been predicted. We need to emphasize here, that we checked the consistency of the predicted model with the data; we did not check the uniqueness of the solution found. We do note that no other model has been either inferred nor proposed, which fits all the same data, but it is quite conceivable that there is such a model, yet to be worked out or published. The approaches by M. Malkov, J.-P. Meyer and L. O'C. Drury, E. Berezho et al., and I. Axford et al. all provide a different approach to the quest for the origin of cosmic rays. The various approaches may yet converge to a common theory.

Focussing now on the concept originally started some time ago, there are a number of important conseqences from the success of the model proposed:

\subsection{A common final state for massive stars}

The are several very interesting consequences from the success of this approach. Some of these consequences follow by necessity, which entails if these consequences can be falsified, then the original model fails. We will identify these critical steps below.

- The abundances in stellar winds near to and beyond 20 solar masses, RSG and WR supergiants dominate the cosmic ray chemical composition - this is in contrast to the superbubble concept; however, since the superbubbles in fact also have a mixture of wind abundances, these two approaches may be consistent in this point with each other. The argument, that the abundances of cosmic rays derive from accelerated dust particles is starkly different, 65.

- The energy in the cosmic ray population of Helium, Carbon, and Oxygen is so large as to require a much larger energy content in cosmic rays per supernova; note that the RSG and WR stars are the dominant contributors, stars which are quite rare. The energy requirement in cosmic rays per supernova of this kind is then about $10^{51}$ ergs.

- If we allow for an overall efficiency of $10 \%$ of putting explosion energy into cosmic rays, the explosion energy is then required to be $10^{52}$ ergs for such supernovae.

- The most important conclusion from the approach outlined here is that 
stars near to or above 25 solar masses explode due to the magnetorotational mechanism. We cannot say for sure at this stage at which exact mass we have a transition in supernova mechanisms to the magnetorotational mechanism, since the cosmic ray argument is really clear only for the WR stars.

- Knee energy and cutoff energy need to be the same for all supernovae contributing. This is easiest if those stars form a "common final state", as regards rotation, magnetic fields, mass, explosive energy.

- There is first evidence for an explosion energy of order $10^{52}$ ergs from a very massive star from SN1998bw, 48, 69. That specific supernova was also highly asymmetric, with a rotational symmetry quite possible, consistent with the mechanism proposed by Bisnovatyi-Kogan.

\subsection{The magneto-rotational mechanism}

The magnetorotational supernova mechanism was suggested by BisnovatyiKogan, 29]. The idea was to get the energy for the explosion from the rotational (gravitational) energy using the magnetic field. Most stars are rotating differentially. The magnetic field is "frozen" into the ionized gas of the star. Then differential rotation leads to an amplification of the toroidal component of the magnetic field. When magnetic pressure becomes comparable with the gas pressure a compression wave forms and moves out to the envelope of the star. When moving along a steeply decreasing density profile this wave transforms to a strong fast MHD shock which pushes part of the envelope of the star to the outside, and so forms an explosion. Simulations of the magnetorotational mechanism for a magnetized cloud [10, 66] show that this mechanism is rather effective. The simulations of the collapse of a rotating pre-supernova star show that after the short collapse stage the star consists of a rapidly rotating core and a slowly rotating envelope. At the transition region between the core and the envelope the rotation is very differential and in such a situation the inclusion of even a weak initial magnetic field could lead to amplification of the toroidal component and so to a magnetorotational explosion. Further work is in [30, 9, 11.

\subsection{Implications for Gamma Ray Bursts?}

There is an interesting resemblance of this explosion mechanism to the concept of a hypernova developed in the context of Gamma Ray Bursts, [70, 73. Using the concept, [75, 76], does indeed suggest an energy scale of also $10^{52} \mathrm{erg}$, derived in that specific model as an upper energy limit implied by a fit to the fluence distribution, allowing for observational selection effects. The recent observations by Schaefer et al., 80, support the concept that at least some GRBs blow up into a stellar wind.

This leads then one more time to the question of what the relation is between Gamma Ray Burst explosions and Supernova explosions, a question explored by many. 
There is much recent work also by C. Wheeler, et al., [2, 3, 49, 94, 95].

\subsection{A bright standard candle for cosmology?}

There is a corollary also for cosmology: If the explosion energy is the same for all supernovae above about a zero age main sequence mass of near 25 solar masses, as suggested by the arguments above, one may speculate even further: Could it be that also the maximum luminosity, or the integral over the luminosity time curve is the same for all such supernovae? There is the obvious problem, that these explosions are rotationally symmetric only, and so present vastly different views to observers at different angles to the symmetry axis. This may be resolved by infrared observations, and also by polarization observations, just as for active galactic nuclei, for which infrared observations give the best approximation for an isotropic emission, and polarization observation do give clues for the angle between symmetry axis and line of sight.

If we could thus derive from observations the maximum luminosity or its time integral, we might have a much brighter standard candle for use in cosmology than the supernovae of type Ia, which derive from the collapse of a white dwarf in a stellar binary system, and have a fairly low explosion energy.

\subsection{Transition: Beyond GZK cutoff}

Let us finish with a brief note on the events beyond the Galaxy, the events with an energy beyond $310^{18} \mathrm{eV}$. Recent reviews are in, e.g., [19, 18. The latest data and their discussions are in [1, 47] for HiRes, and in [87, 88, 44, 53, 97, 98. for AGASA.

The main dispute at the present time is the apparent discrepancy between the AGASA data and the HiRes data, with the HiRes data suggesting a downturn of the overall spectrum in qualitative agreement with the GZK-cutoff concept, although at a somewhat higher energy, as would be expected from a highly inhomogeneous source distribution, such as any subpopulation of galaxies. On the other hand, the AGASA data suggest a continuation of the spectrum to higher energies, which would require a new component to appear from under the lower flux contributors, in perfect agreement with a scenario involving the decay of topological defects. The real discrepancy between the two data sets is only about 2 sigma, as discussed at the meeting in Paris June 2002. It is widely expected that AUGER data will resolve this issue, and then maybe the further experiments will be required, such as EUSO and OWL, the European and American space missions to detect airshowers at extremely high energies.

\section{Conclusions}

The successful fit of the new KASCADE data, under the constraint to also fit the higher energy data from HiRes, AGASA and Yakutsk, with the cosmic ray

source model originally proposed some time ago, and developed further since 
then, leads to a number of important conclusions. The most important points are:

- The origin of Galactic cosmic rays may be much closer to a full understanding of their origin. Sofar all quantitative tests, which have been made, show consistency with the model proposed earlier. But much work remains to be done.

- All stars above a zero age main sequence mass of about 25 solar masses converge to a "common final state".

- Those stars explode with about $10^{52}$ ergs in a highly anisotropic explosion, following the mechanism originally proposed by G. Bisnovatyi-Kogan more than 30 years ago, which was then based on a broader suggestion by Kardashev, involving rotation, magnetic fields and potential energy.

- These supernovae may constitute a new very bright standard candle, useful in cosmology, provided we could determine their luminosity integrated over $4 \pi$ from infrared, polarization or other observations.

\section{Acknowledgements}

P.L. Biermann would like to acknowledge the hospitality, first at the University of Maryland, offered by his colleague Eun-Suk Seo in the early spring of 2002, and then during his prolonged stay at the University of Paris VII, in the late spring 2002, offered by his colleagues Norma Sanchez and Hector de Vega, as well as their hospitality at many meetings in Erice, Paris and Palermo. PLB would especially like to acknowledge a day-long debate with I. Axford at the Taiwan meeting in April 2002, arguing about the agreements and also differences between the superbubble concept and the stellar wind concept. PLB would also like to express his appreciation to N. Langer for a similarly long discussion at Utrecht in May 2002, arguing about supernova physics. And, PLB would also like to acknowledge many discussions with G. Schatz on the intricacies of trying to elucidate cosmic ray spectra from shower data. The material in this paper was the topic of a long helpful debate with T. Stanev in Paris in the spring of 2002, for which PLB is also very grateful. Help with some of the data was generously supplied by Zh. Cao from Utah and D. Bergman from Rutgers. The authors would like to acknowledge with gratitude discussions with many friends and colleagues, especially E.J. Ahn, I. Axford, Zh. Cao, S. Casanova, M. Chirvasa, A. Donea, R. Engel, T. Enßlin, H. Falcke, C. Galea, R. Engel, A. Haungs, H. Kang, M. Kaufman, P.P. Kronberg, N. Langer, A. Lazarian, H. Lee, K. Mannheim, S. Markoff, G. Medina-Tanco, A. Meli, F. Munyaneza, A. Olinto, B. Nath, G. Pavalas, A. Popescu, R. Protheroe, G. Pugliese, J. Rachen, W. Rhode, G. Romero, E. Roulet, D. Ryu, N. Sanchez, K. Sato, G. Schäfer, G. Schatz, H. Seemann, E.-S. Seo, R. Sina, T. Stanev, V. Tudose, H. de Vega, Y.P. Wang, A. Watson, T. Weiler, St. Westerhoff, and Ch. Zier. Work with PLB is being supported through the AUGER theory and membership grant 05 CU1ERA/3 through DESY/BMBF (Germany); further 
support for the work with PLB comes from the DFG, DAAD, Humboldt Foundation (all Germany), grant 2000/06695-0 from FAPESP (Brasil) through G. Medina-Tanco, a grant from KOSEF (Korea) through H. Kang and D. Ryu, a grant from ARC (Australia) through R.J. Protheroe, and European INTAS/ Erasmus/ Sokrates/ Phare grants with partners V. Berezinsky, M.V. Rusu, and V. Ureche. All these sources of support are gratefully acknowledged. The work reported here was also specifically supported by the DAAD through a fellowship to S. Ter-Antonyan.

\section{References}

[1] Abu-Zayyad, , et al., (2002), astro-ph/0208243

[2] Akiyama, Sh., Wheeler, J.C., Meier, D.L., Lichtenstadt, I., Astrophys. J. (in press) (2002), astro-ph/0208128

[3] Akiyama, Sh., Wheeler,J.C., in proc. "Core Collapse of Massive Stars, ed. C. L. Fryer, Kluwer Academic Publ., (2002), astro-ph/0211458

[4] Antoni, T., et al., Astropart.Phys. 14, 245 (2001)

[5] Antoni, T., et al., Astropart.Phys. 16, 373-386 (2002)

[6] Antoni, T., et al., Astropart.Phys. 16, 245-263 (2002)

[7] Antoni, T., et al., Astropart.Phys. 18, 319-331 (2003)

[8] Apananseko, A.V., et al., Proc. 26th ICRC, Salt Lake City 3, 163 (1999)

[9] Ardeljan, N.V., Bisnovatyi-Kogan, G.S., Kosmachevskii, K.V., Moiseenko, S.G., Astron. 85 Astrophys. ASuppl. 115 , 573 (1996)

[10] Ardeljan, N.V., Bisnovatyi-Kogan, G.S., Moiseenko, S.G., Astron. \& Astroph. 355, 1181 - 1190 (2000)

[11] Ardeljan, N.V., Bisnovatyi-Kogan, G.S., Moiseenko, S.G., in Proc. of XX Texas Symposium of Relativistic astrophysics. Austin,AIP Conf. Proc. 586, 433 (2001)

[12] Asakimory, K. et al., Astrophys. J. 502, 278 (1998)

[13] Axford, W. I., Astrophys. J. Suppl. 90, 937 - 944 (1994)

[14] Avakyan, V.V., et al., Yadernaya Fizika 50, 134 (1989), Soviet Journal of Nuclear Physics, in Russian)

[15] Baade, W., Zwicky, F., 1934, Proc. Nat. Acad. Science, 20, no. 5, 259 263.

[16] Bell, A.R., 1978 Month. Not. Roy. Astr. Soc. 182, 147 - 156, and 443 455. 
[17] Berezinskii, V.S., et al., "Astrophysics of Cosmic Rays", North-Holland, Amsterdam (especially chapter IV) (1990).

[18] Bhattacharjee, P. \& Sigl, G., Physics Reports, 327, 109 - 247 (2000), astro-ph/9811011

[19] Biermann, P.L., 1997, Journal of Physics G 23, 1.

[20] Biermann, P.L., Astron. $E$ Astroph. 271, 649 (1993), astro-ph/9301008.

[21] Biermann, P.L. \& and Cassinelli, J.P., Astron. \& Astroph. 277, 691 (1993), astro-ph/9305003.

[22] Biermann, P.L., \& Strom, R.G., Astron. \& Astroph. 275, 659 (1993), astro-ph/9303013

[23] Biermann, P.L., invited plenary lecture at the 23rd International Conference on Cosmic Rays, in Proc. "Invited, Rapporteur and Highlight papers"; Eds. D. A. Leahy et al., World Scientific, Singapore, 1994, p. 45

[24] Biermann, P.L., in Cosmic winds and the Heliosphere, Eds. J. R. Jokipii et al., Univ. of Arizona press, p. 887 - 957 (1997), astro-ph/9501030.

[25] Biermann, P.L., review at the Vulcano meeting on "Frontier objects in astrophysics and particle physics", Eds. Giovannelli et al., Italian Physical Society, Conf. Proc. 47, Bologna 1995, p. 469

[26] Biermann, P.L., some concluding remarks at the Vulcano meeting on Frontier objects in astrophysics and particle physics, Eds. Giovannelli et a., Italian Physical Society, Conf. Proc. 47, Bologna 1995, p. 593

[27] Biermann, P.L., invited lecture at the Nuclear Astrophysics meeting at Hirschegg, 1997, in Proc., GSI, Darmstadt, p. 211 - 222, 1998

[28] Biermann, P.L., Langer, N., Seo, E.-S., Stanev, T., Astron. \& Astroph. 369, 269 - 277 (2001)

[29] Bisnovatyi-Kogan, G.S., Astron.Zh. (Sov. Astron.), 47, 813 (1970)

[30] Bisnovatyi-Kogan, G.S., Moiseenko, S.G., Sov. Astron. 36 285, (1992)

[31] Blandford, R. D.; Ostriker, J. P., Astrophys. J. Letters 221, L29 - L32 (1978)

[32] Chilingaryan, A.A., et al., Proc. 26th ICRC, (Salt Lake City) 1, 240 (1999)

[33] Drury, L. O'C., Rep. Progr. Phys. 46, 973 - 1027 (1983)

[34] Fermi, E., Phys. Rev. 2nd ser., 75, no. 8, 1169 - 1174 (1949)

[35] Fermi, E., Astrophys.J. 119, 1 - 6 (1954) 
[36] Fletcher, R.S. , Gaisser, T.K. , Lipari, P. , \& Stanev, T. , Phys.Rev. D 50, 5710 (1994) / Engel, J., Gaisser, T.K. , Lipari, P. , Stanev, T. , Phys.Rev.D. 46, 5013 (1992) / Engel, R., et al., Proc. 26th ICRS (Salt Lake City), 1, 415 (1999)

[37] Gaisser, T.K., Cosmic Rays and Particle Physics, Cambridge Univ. Press (1990)

[38] Garcia-Munoz, M., Mason, G. M., Simpson, J. A., Astrophys. J. 217, 859 - 877 (1977)

[39] Garcia-Munoz, M., et al., Astrophys. J. Suppl. 64, 269 - 304 (1987)

[40] Ginzburg, V.L. \& Syrovatskii, S.I., The origin of cosmic rays, Pergamon Press, Oxford (1964), Russian edition (1963).

[41] Glasstetter, R., et al., Proc. 26th ICRC Salt Lake City 1222 (1999)

[42] Glasstetter, R., et al., Nucl.Phys. B (Proc.Suppl) 75A, 238 (1999)

[43] Haungs, A. 18th ECRS, Moscow (2002) Ep1a.2,/ http://dbserv.npi.msu.su/ 18sym/conference/transparencies.phtml/ K.-H. Kampert et al. (KASCADE collaboration), Highlight Paper, Proc. 27th ICRC, Hamburg (2001), astro-ph/0204205 (2002)

[44] Hayashida, N., et al., (2000), astro-ph/0008102, appendix for Takeda et al.., Astrophys. J. 522, 225 - 237 (1999)

[45] Heck, D., Knapp, J., Capdevielle, J.N., Schatz, G., \& Thouw, T., Forschungszentrum Karlsruhe Report, FZKA Nr. 601990 p. (1998)

[46] Hillas, A. M., Ann. Rev. Astron. Astrophys. 22, 425 (1984).

[47] HiRes-Coll., Astropart. Phys. (submitted) (2002), astro-ph/0208301

[48] Höflich, P., Wheeler, J. C., Wang, L., Astrophys. J. 521, 179 - 189 (1999)

[49] Höflich, P., et al., in Proc. IAU Symposium 212 on Massive Stars, D. Reidel Conf. Series, ed. E. van den Hucht, (2002), astro-ph/0207272

[50] Hoerandel, J.R., et al., Proc. 27th ICRC, Hamburg, 1, 71 (2001)

[51] Hoerandel, J.R., Astropart. Phys. (in press) (2002), astro-ph/0210453

[52] Hunter, S. D., et al., Astrophys. J. 481, 205 (1997)

[53] Ide, Y., et al., Publ. Astron. Soc. Japan 53, 1153 - 1162 (2001)

[54] Jokipii, J. R., Astrophys. J. 313, 842-846 (1987)

[55] Kalmykov, N.N., \& Ostapchenko, S.S., Yad. Fiz. 56105 (1993); Phys.At.Nucl. 56, 346 (1993)

[56] Kardashev, N. S., Astronomicheskii Zhurnal 41, 807 (1964)

[57] Kronberg, P.P., Rep. Prog. Phys., 57, 325 - 382 (1994) 
[58] Krymskii, G. F., Akademiia Nauk SSSR, Doklady 234, 1306 - 1308 (1977), in Russian

[59] Kulsrud, R.M., Annual Rev. of Astron. E Astrophys. 37, 37 (1999)

[60] Lagage, P. O., \& Cesarsky, C. J., Astron. \& Astroph. 125, 249-257 (1983)

[61] Learned, J.G. \& Mannheim, K., Ann. Rev. Nucl. \& Part. Sci. 50, 679 $749(2000)$

[62] Malkov, M. A., O'C Drury, L., Rep. on Progr. in Phys. 64, 429 - 481 (2001)

[63] Mezger, P. G., Duschl, W. J., Zylka, R., Astron. \& Astroph. Rev. 7, 289 - 388 (1996)

[64] Melia, F, Falcke, H., Annual Rev. of Astron. 83 Astrophys. 39, p. 309-352 (2001)

[65] Meyer, J.-P., Drury, L. O’C., Ellison, D. C., ApJ 487, 182 (1997)

[66] Moiseenko, S.G., Ardeljan, N.V., Bisnovatyi-Kogan, G.S., Rev. Mex. Astron. ES Astroph., (Serie de Conferencias), 15, 231 - 233 (2003)

[67] Mori, M., Astrophys. J. 478, 225 (1997)

[68] Nagano, M., Watson, A.A., Rev. Mod. Phys., 72, 689 - 732 (2000).

[69] Nakamura, T., Mazzali, P. A., Nomoto, K., Iwamoto, K., Astrophys. J. 550, 991 - 999 (2001)

[70] Paczynski, B., in Proc. Gamma-Ray Burst: 4th Huntsville Symposium, Huntsville, AL Sept. 1997. Eds. by Ch. A. Meegan et al.. Woodbury, New York : AIP conference proceedings ; 428, p.783 (1998)

[71] Peters, B., Nuovo Cimento Suppl., XIV, 436 - 456 (1959)

[72] Peters, B., Nuovo Cimento, XXII, 800 - 819 (1961)

[73] Piran, T., Physics Reports 314, 575 (1999)

[74] Ptuskin, V.S. et al., ICRC 26, ms. OG 3.2.32, vol. 4, p. 291 (1999)

[75] Pugliese, G., Falcke, H., Biermann, P.L., Astron. $E$ Astroph. Letters 344, L37 - L40 (1999), astroph/9903036

[76] Pugliese, G., Falcke, H., Wang, Y., Biermann, P.L., Astron. \& Astroph. 358, 409 - 416 (2000), astro-ph/0003025

[77] Rachen, J.P., \& Biermann, P.L., Astron. \& Astroph. 272, 161 (1993), astro-ph/9301010

[78] Rachen, J.P., Stanev, T., \& Biermann, P.L., Astron. \& Astroph. 273, 377 (1993), astro-ph/9302005 
[79] Ratkiewicz, R., Axford, W. I., \& McKenzie, J. F., Astron. \& Astroph. 291, 935 - 942 (1994)

[80] Schaefer, B.E., et al., Astrophys. J. (submitted) (2002), astro-ph/0211189

[81] Schatz, G., Astropart. Phys. 17, 13-22 (2002)

[82] Seemann, H. \& Biermann, P.L., Astron. \& Astroph. 327, 273 (1997), astro-ph/9706117.

[83] Silberberg, R., \& Tsao, C. H., Astrophys. J. Letters 352, L49 - L52 (1990)

[84] Snowden, S.L., et al., Astrophys. J. 485, 125 (1997)

[85] Stanev, T., Biermann, P.L., \& Gaisser, T.K., Astron. $\&$ Astroph. 274, 902 (1993), astro-ph/9303006

[86] Swordy, S.P., Fortson, L.F., et al., Astropart. Phys. 18, 129 (2002), astro-ph/0202159

[87] Takeda, M., et al., Phys. Rev. Letters 81, 1163 - 1166 (1998)

[88] Takeda, M., et al., Astrophys. J. 522, 225 - 237 (1999)

[89] Ter-Antonyan, S.V., \& Haroyan, L.S., Proc. of the workshop ANI98, Forschungszentrum Karlsruhe, FZKA 6216, 115 (1998)

[90] Ter-Antonyan, S.V. , \& Haroyan, L.S., (2000) hep-ex/0003006

[91] Ter-Antonyan, S.V., \& Biermann, P.L., astro-ph/0106076 (2001)

[92] Ulrich, H., et al., Proc. 27th ICRC, Hamburg, 1, 97 (2001)

[93] Völk, H. J. \& Biermann, P. L., Astrophys. J. Letters 333, L65 - L68 (1988)

[94] Wang, L., et al., Astrophys. J. 579, 671 - 677 (2002), astro-ph/0205337

[95] Wheeler, J.C., Am.J.Phys. 71, 11 - 22 (2003), astro-ph/0209514

[96] Wiebel-Sooth, B., Biermann, P.L., Landolt-Börnstein, vol. VI/3c, Springer Verlag, p. 37 - 90 (1999)

[97] Yoshiguchi, H., Nagataki, S., Tsubaki, S., Sato, K., Astrophys. J. , (in press) (2002), astro-ph/0210132

[98] Yoshiguchi, H., et al., Publ. Astron. Soc. Japan , (in press) (2002), astro-ph/0212061 